\title{
A New Chiral Catalytic Ceramic Membrane Reactor and Its Use in Catalyzing Epoxidation of Styrene
}

\author{
Min LIU ${ }^{1, \text { a }}$, Zhi-Ping Zhao*,1, b , Xiao-Lan Wang ${ }^{1}$ \\ ${ }^{1}$ School of Chemical Engineering and the Environment, Beijing Institute of Technology, Beijing, \\ 100081, China \\ aemail: stkxliumin2010@163.com, bemail: zhaozp@bit.edu.cn( ${ }^{*}$ corresponding author)
}

\author{
Keywords: Salen-Mn Catalyst; Membrane Reactor; Epoxidation
}

\begin{abstract}
A new chiral salen-Mn catalytic ceramic membrane reactor was constructed by two steps. Firstly, the ceramic membrane was modified by grafting 3-aminopropyltriethoxysilane. Secondly, the Jacobsen type salen-Mn(III) catalyst was immobilized onto the modified ceramic membranes through chemical bonding of metal centers of the catalyst and amino-groups on the membrane. The catalytic performances of the reactor were investigated by asymmetric epoxidation of styrene, and $25.2 \%$ conversion rate and $31.6 \%$ ee value were obtained while the mol ratio of catalyst/styrene was $0.121 \%$. The stability of the obtained reactor was tested by catalyzing four runs of epoxidation reactions. The reactor can reuse the catalyst directly without any separation operation.
\end{abstract}

\section{Introduction}

Chiral epoxides are important building blocks for synthesizing a variety of valuable chiral compounds such as pharmaceuticals and agrochemicals through regioselective ring-opening reactions [1][2]. The asymmetric epoxidation of unfunctionalized olefins catalyzed by chiral salen-Mn catalyst is one of the effectual methods to prepare the chiral epoxides since the catalyst is of excellent catalytic properties [3][4]. However, the catalyst is expensive, and it is difficult to separate the catalyst from the reaction mixture to realize recycling, so many reported works focused on the immobilization of the catalyst in order to resolve this problem [5].

The supports for immobilizing catalyst involve organic and inorganic ones. Inorganic matrixes had received much attention [6][7] in recent years due to their high chemical stability. The mostly used inorganic supports for immobilizing salen catalysts are clay [8], activated silica [9], molecular sieve [10] and so on. But the separation of immobilized-catalysts in suspension is also a challenge in industry field applications, especially for ultrafine particles [11]. Besides, the supported catalysts cannot be distributed well in the reaction system, which has a negative influence on mass transfer. Such problems can be avoided effectively if the catalyst molecules are immobilized in or on membranes [12].

In our previous work [13], mono-chloromethyl-salen-Mn catalyst was immobilized onto ceramic membrane. The salen-Mn immobilized membrane was packed as a reactor (Fig 1) and used in catalyzing epoxidation of styrene. The reactor realized catalyst recycle without separation operations. But the Jacobsen catalyst with four tert-butyl groups was reported to be of most excellent catalytic properties among kinds of salen-Mn catalysts. And it only needs three steps to synthesize the tetra-tert-butyl-salen-Mn(III) catalyst, which is much simpler than the way of synthesizing mono-chloromethyl-salen-Mn catalyst.

In this paper a new chiral salen-Mn catalytic ceramic membrane reactor was constructed by attached Jacobsen catalyst to amino-modified ceramic membrane. The catalytic performances of the reactor were investigated by catalyzing the asymmetric epoxidation of styrene.

\section{Construction of Salen-Mn Membrane Reactor}

First, Jacobsen type salen-Mn catalyst with four tert-butyl groups was synthesized by three steps (Fig 2, a) according to the method of literature [13]. Second, ceramic membrane (membrane layer: 
$\mathrm{ZrO}$, supporting layer: $\mathrm{Al}_{2} \mathrm{O}_{3}$, mean pore size: $70 \mathrm{~nm}, 19$ channels) was activated by $1 \%(\mathrm{w} / \mathrm{w})$ diluted hydrochloric acid solution and assembled as a reactor showed in Fig 1 after being dehydrated under vacuum. Toluene $(150 \mathrm{ml})$ and 3-aminopropyltriethoxysilane $(2 \mathrm{ml})$ were added into the feed tank under the protection of $\mathrm{N}_{2}$, and the reaction mixture was cycled at room temperature for 48 hours. When finished, the reaction system was washed by fresh toluene. Third, salen-Mn catalyst $(1 \mathrm{~g})$, anhydrous potassium carbonate $(0.6 \mathrm{~g})$ and toluene $(60 \mathrm{ml})$ were added into the reactor, the reaction solution was cycled at $80^{\circ} \mathrm{C}$ for 20 hours. The system was washed by fresh toluene after cooling down to room temperature until the solution was colorless. The membrane was washed by distilled water until neutral and dehydrated under vacuum at $120^{\circ} \mathrm{C}$. The immobilization degree of the catalyst was tested by ultraviolet spectrophotometric method. The grafting and immobilizing routes are showed in Fig 2 (b).

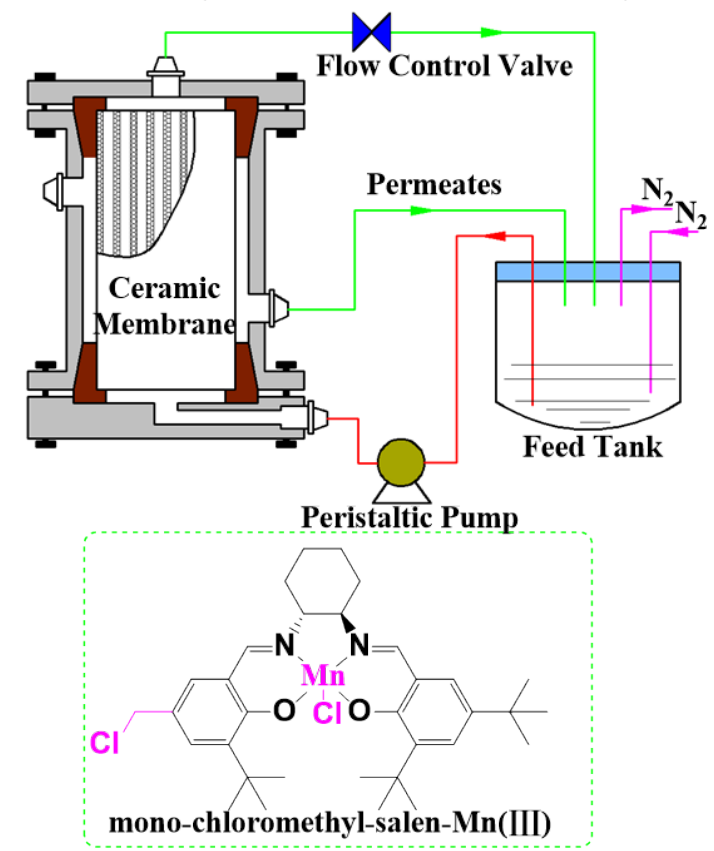

Fig.1. Scheme of ceramic membrane reactor and the structure of mono-chloromehyl-salen- $\mathrm{Mn}^{[13]}$
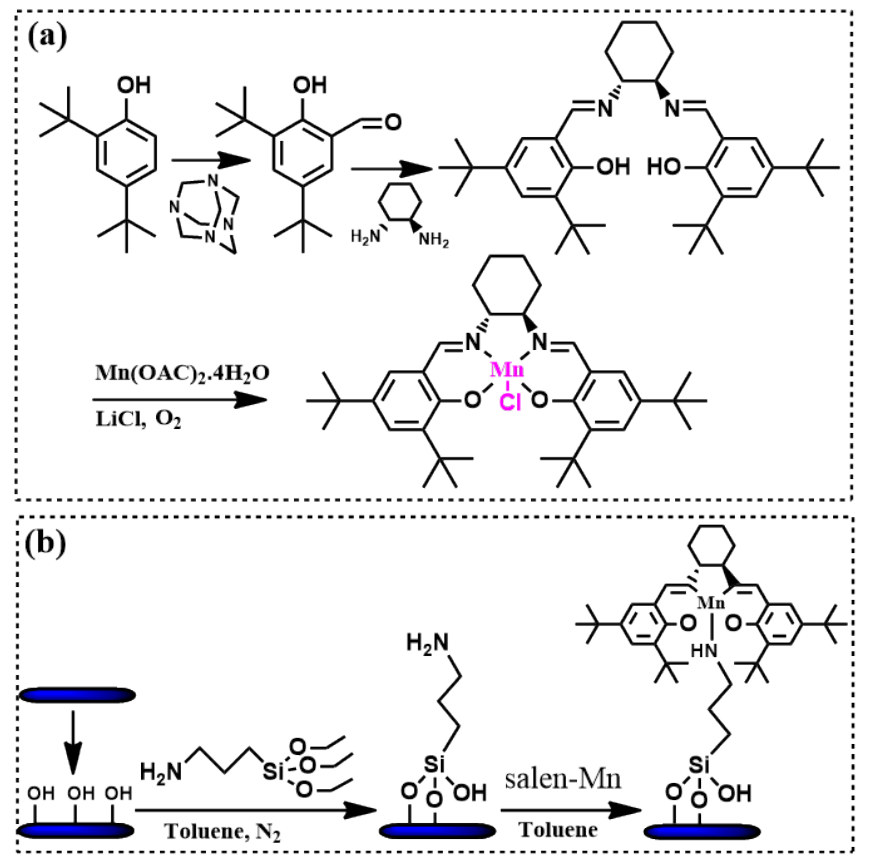

Fig. 2. Routes of synthesis of tetra-tert-butyl-salen-Mn catalyst and immobilizing the catalyst onto ceramic membrane

\section{Asymmetric Epoxidation of Styrene by Membrane Reactor}

Styrene (4 mmol), 4-methylmorpholine (20 mmol), toluene (0.2g, as internal standard substance) and dichloromethane $(55 \mathrm{ml})$ were added into the feed tank, the mixture was cooled to $0{ }^{\circ} \mathrm{C}$ and cycled for $15 \mathrm{~min}$, then 3-chloroperbenzoic acid was added and the reaction mixture was cycled for 11 hours. The reaction was detected periodically by GC (TECHCOMP 7890II) with a chiral $\beta$-cyclodextrin capillary column (RESTEK RT-BetaDEXse, $30 \mathrm{~m} \times 0.25 \mathrm{~mm} \times 0.25 \mu \mathrm{m}$ ). The reaction formula is showed in Fig 3.<smiles>C(C=Cc1ccc(C2O[C@H]2c2ccccc2)cc1)=Cc1ccccc1</smiles>

Fig. 3. Epoxidation reaction of styrene 


\section{Results and Discussion}

The results of catalytic reaction are showed in Table 1 . Under the given reaction conditions, the mol ratio of the immobilized salen-Mn catalyst/styrene was $0.121 \%$. It can be found that the immobilization degree of tetra-tert-butyl-salen-Mn catalyst is slightly lower than mono-methly-salen-Mn catalyst $(0.129 \%)$ [13] under the same immobilization conditions. But comparable catalytic efficiency (TOF value) was obtained in contrast to mono-methly-salen-Mn ceramic membrane reactor. From the data one can find that the conversion rate of styrene was increased with the increase of reaction time, but the growth trend was not so obvious after 9 hours. And the TOF values of the catalyst were decreased with the reaction time. It because that the concentration of styrene decreased with the proceeding of the reaction, so the contact chance of styrene with catalytic center was reduced. The slightly low conversion rate may be attributed to the low immobilization degree of the tetra-tert-butyl-salen-Mn catalyst. And the tetra-tert-butyl-salen-Mn catalyst was attached to the support through the metal center by which the distance of the catalytic center with the support was relatively short. So, it may have a negative influence on the reactivity and enantioselectivity of the catalyst. The further research should focus on improving immobilization degree of the catalyst through optimizing the grafting and immobilizing reaction conditions, and adopting silane coupling agent with longer carbon chain.

Table 1 The catalytic performances of membrane reactor for catalyzing epoxidation of styrene

\begin{tabular}{ccccc}
\hline Time (h) & Conv. (\%) & ee (\%) & TON $^{\mathrm{a}}$ & $\mathrm{TOF}^{2}\left(10^{-2} \mathrm{~S}^{-1}\right)^{\mathrm{b}}$ \\
\hline 2 & 15.6 & $31.5(\mathrm{R})$ & 84.8 & 1.1771 \\
3 & 16.6 & $31.7(\mathrm{R})$ & 90.3 & 0.8365 \\
4 & 16.9 & $31.3(\mathrm{R})$ & 91.7 & 0.6369 \\
5 & 18.1 & $31.1(\mathrm{R})$ & 98.1 & 0.5448 \\
7 & 22.2 & $31.6(\mathrm{R})$ & 120.7 & 0.4792 \\
8 & 23.1 & $32.1(\mathrm{R})$ & 126.1 & 0.4378 \\
9 & 24.7 & $31.9(\mathrm{R})$ & 134.6 & 0.4154 \\
11 & 25.2 & $31.6(\mathrm{R})$ & 137.1 & 0.3461 \\
\hline \multirow{2}{*}{ TON=mol ratio of product/catalyst; ${ }^{\mathrm{b}}$ TOF=TON/reaction time (s) }
\end{tabular}

In order to investigate the stability of the membrane catalyst, the reactor was used for catalyzing the epoxidation of styrene for 4 cycles. After each reaction cycle, the reactor was washed by fresh dichloromethane and used directly to the next cycle without unloading the membrane. The tested results were showed in Table 2. The conversion rate and ee value decreased slightly after each reaction cycle. It may be because that part of the catalysts dropped off during the epoxidation reactions. 
Table 2 The testing results of stability of tetra-tert-butyl-salen-Mn(III) ceramic membrane reactor

\begin{tabular}{cccccc}
\hline $\begin{array}{c}\text { Run } \\
\text { numeber }\end{array}$ & $\begin{array}{c}\text { Time } \\
(\mathrm{h})\end{array}$ & $\begin{array}{c}\text { Conv. } \\
(\%)\end{array}$ & $\begin{array}{c}\text { ee } \\
(\%)\end{array}$ & TON & $\begin{array}{c}\text { TOF } \\
\left(10^{-2} \mathrm{~S}^{-1}\right)\end{array}$ \\
\hline 1 & 9 & 24.7 & $31.9(\mathrm{R})$ & 134.6 & 0.4154 \\
2 & 9 & 23.1 & $31.4(\mathrm{R})$ & 125.4 & 0.3871 \\
3 & 9 & 20.5 & $30.4(\mathrm{R})$ & 110.4 & 0.3409 \\
4 & 9 & 17.4 & $27.7(\mathrm{R})$ & 91.8 & 0.2834 \\
\hline
\end{tabular}

\section{Conclusion}

A chiral catalytic membrane reactor was constructed by immobilizing tetra-tert-butyl-salen-Mn catalyst onto amino-grafted ceramic membrane. The catalytic efficiency of the obtained reactor was comparable to mono-chloromethyl-salen-Mn ceramic membrane reactor. The catalytic properties of the membrane reactor showed slightly decreased during four runs of epoxidation of styrene. And the membrane reactor can recycle the catalyst without separate operations.

\section{Acknowledgement}

In this paper, the research was supported by the National Natural Science Foundation of China (20676015) and Research Fund for the Doctoral Program of Higher Education of China (No.20131101130005).

\section{References}

[1] Zhang, H.; Li, C. Asymmetric epoxidation of 6-cyano-2,2-dimethylchromene on Mn(salen) catalyst immobilized in mesoporous materials. Tetrahedron. 2006, 62, 6640-6649.

[2] Yu, K.; Gu, Z. C.; Ji, R. N.; Lou, L. L. Effect of pore size on the performance of mesoporous material supported chiral Mn(III) salen complex for the epoxidation of unfunctionalized olefins. J. Catal. 2007, 252, 312-320.

[3] Zou, X. C.; Shi, K.; Wang, C. Chiral MnIII(Salen) supported on tunable phenoxyl group modified zirconium poly (styrene-phenylvinylphosphonate)-phosphate as an efficient catalyst for epoxidation of unfunctionalized olefins. Chinese. J. Catal. 2014, 35, 1446-1455.

[4] Baleizão, C.; Garcia, H. Chiral salen complexes: An overview to recoverable and reusable homogeneous and heterogeneous catalysts. Chem. Rev. 2006, 106, 3987-4043.

[5] Huang, J.; Cai, J. L.; Li, C. M.; Fu, X. K. Immobilized chiral Mn (III) salen-containing oniumsalt onto ZnPS-PVPA for asymmetric epoxidation of unfunctionalized olefins. Inorg. Chem. Commun. 2014, 44, 20-24.

[6] Kureshy, R. I.; Ahmad, I.; Khan, N. H.; Abdi, S. H. R.; Singh, S.; Pandia, P. H.; Jasra, R. V. New immobilized chiral $\mathrm{Mn}(\mathrm{III})$ salen complexes on pyridine $\mathrm{N}$-oxide-modified MCM-41 as effective catalysts for epoxidation of nonfunctionalized alkenes. J. Catal. 2005, 235, 28-34.

[7] Lou, L. L.; Yu, K.; Ding, F.; Peng, X.; Dong, M.; Zhang, C.; Liu, S. Covalently anchored chiral $\mathrm{Mn}(\mathrm{III})$ salen-containing ionic species on mesoporous materials as effective catalysts for asymmetric epoxidation of unfunctionalized olefins. J. Catal. 2007, 249, 102-110.

[8] Kuźniarska-Biernacka, I.; Silva, A. R.; Carvalho, A. P.; Pires, J.; Freire, C. Anchoring of chiral manganese(III) salen complex onto organo clay and porous clay heterostructure and catalytic activity in alkene epoxidation. Catal. Lett. 2010, 134, 63-71. 
[9] Zhang, H. D.; Xiang, S.; Xiao, J. L.; Li, Can. Heterogeneous enantioselective epoxidation catalyzed by $\mathrm{Mn}$ (salen) complexes grafted onto mesoporous materials by phenoxy group. J. Mol. Catal A. Chem. 2005, 238, 175-184.

[10] Lou, L. L.; Jiang, S.; Yu, K.; Gu, Z. C.; Ji, R. N.; Dong, Y. L.; Liu, S. X. Mesoporous silicas functionalized with aminopropyl via co-condensation: Effective supports for chiral Mn(III) salen complex. Micropor. Mesopor. Mat. 2011, 142, 214-220.

[11] Lu, C. J.; Chen, R. Z.; Xing, W. H.; Jin, W. Q.; Xu, N. P. A submerged membrane reactor for continuous phenol hydroxylation over TS-1. AIChE. J. 2008, 54, 1842-1849.

[12] Chen, R. Z.; Jiang, Y. G.; Xing, W. H.; Jin, W. Q. Fabrication and catalytic properties of palladium nanoparticles deposited on a silanized asymmetric ceramic support. Ind. Eng. Chem. Res. 2011, 50, 4405-4411.

[13] Liu, M.; Zhao, Z. P.; Chen, K. C.; Liu, W. F. New chiral catalytic membrane reactor created by immobilizing salen-Mn(III) onto APTES modified ceramic membrane and its performances in epoxidation of styrene. Catal. Commun. 2015, 64, 70-74. 